\title{
Multi-hump soliton-like structures in interactions of lasers and Bose-Einstein condensates
}

\author{
F. Cattani ${ }^{1}$, A. Kim ${ }^{2}$, T. Hansson ${ }^{3}$, D. Anderson ${ }^{3}$ and M. LisaK $^{3}$ \\ 1 School of Mathematics, University of Southampton, Southampton, SO17 1BJ, UK \\ 2 Institute of Applied Physics, Russian Academy of Sciences - 603950 Nizhny Novgorod, Russia \\ 3 Department of Radio and Space Science, Chalmers University of Technology - SE-412 96 Göteborg, Sweden
}

PACS $37.10 . \mathrm{Vz}$ - Mechanical effects of light on atoms, molecules, and ions

PACS 42.65.Sf - Dynamics of nonlinear optical systems

PACS 03.75.Be - Atom optics

\begin{abstract}
An investigation is made of multihump and periodic solutions of the semiclassical coupled equations describing laser radiation copropagating with a Bose-Einstein condensate. Solutions reminiscent of optical vector solitons have been found and have been used to gain understanding of the dynamics observed in the numerical simulations, in particular to shed light on the phenomenon of jet emission from a condensate interacting with a laser.
\end{abstract}

Introduction. - The experimental realisation of Bose-Einstein condensates (BEC) and of coherent atomic beams has inspired a renewed effort in the theoretical modelling of such physical systems. Of particular interest from the point of view of applications, is the possibility of manipulating atomic beam structures by using their interactions with light. Besides, due to the mathematical modelling of these interactions, these phenomena can also offer a possible test of the analogies between optics and quantum matter waves, which is what we would like to explore further in this work.

It is well known that the dynamics of a BEC satisfies an equation which has the same mathematical structure of the nonlinear Schrödinger equation, the fundamental equation of nonlinear optics, [1], the Kerr-like nonlinearity being due to the atom-atom interactions which can be either focusing or defocusing depending on the sign of the scattering length. Considering the interactions of a $\mathrm{BEC}$ with laser radiation brings new terms into the atom equation whose form depends on the modelling of the interactions, the simplest form being the dipole-dipole one. Saffman started to study the consequences of these interactions, [2] while Krutistky et al. derived a fundamental model from first principles within the framework of quantum field theory and later generalized it to consider atom transitions (in the case of high atom densities), [3]. More recently, the same structure for the atom equation was derived within a semi-classical approach, [4]. The idea there was to work out an expression for the force exerted by light on atoms from basic classical physics and use it as a potential term in the Schrödinger equation of the atoms. It is important to notice that, within the limits of a semi-classical approach, the atom equation agrees with the more general equation of [3]. The point is that a natural consequence of BEC atoms interacting with photons is the emergence of a coupling between the dynamics of the atoms and that of the laser radiation. In fact, what these models describe is photons from the incident radiation exciting atoms which in turn re-emit photons, which are then absorbed by other atoms, thus giving rise to a long-range interatomic interaction, $[3,5]$. This obviously has an effect not only on the atoms but also on the radiation. This gives rise to a coupling term in the atom equation and the need for an additional equation describing also the dynamics of the radiation. It is this coupling that can bring about novel effects. It has been shown via numerical simulation of the coupled dynamics that selflocalised structures and mutual atom-light guiding can be achieved exploiting the dipole-dipole interactions, [6]. In particular it was observed that solitary-like localised structures can be generated and emitted from a central bunch of atoms, [7]. This is suggestive of analogous optical effects such as soliton ejection, see [8] and references therein, and we will try here to obtain an understanding of how the structures ejected in laser-BEC interactions are created and emitted. The peculiarity of the emission 
discussed here is that the generation of the solitary-like structures is entirely due to the atom-laser coupling and their ejection from the region where they have been created can be ascribed to their tail-tail interaction. This sort of interaction, being the structures initially well separated, is determined by the overlap of the structures themselves, i.e. the cross terms arising when calculating terms such as $\left|\psi_{T O T}\right|^{2}$ if $\psi_{T O T}$ can be written approximately as the sum of two or more localized functions. Since these functions are assumed to be spatially separated, the only contributions to the interaction term $\left|\psi_{T O T}\right|^{2}$ will come from their overlapping tails.

In optics, for example in the early study of Gubbels et al., [9], up to the more recent work of Assanto et al., [10], the emission is engineered in such a way that the trap or guide does not undergo any modification. Similarly in studies of soliton emission from BECs, see for example [11], the basic physical mechanism for emission concerns the atom-atom interactions and the trap is not modified. In our case, the atoms modify the medium through which they propagate, i.e. the laser radiation, which in turn affects the atom propagation via a nonlinear coupling. If mutual trapping can be achieved, localised symbiotic atom-light structures will start to propagate together and the emission will be due to the interaction of these newly born structures rather than to tunneling or engineering of the trap. This process is also reminiscent of the solitonic gluons studied by Ostrovskaya et al., [12].

Inspired by numerical simulations which clearly show how, during the coupled propagation of laser and atoms, symmetric and mutually localised structures are formed and then ejected, the first step we would like to take is an investigation into the possible existence of multi-hump stationary solutions of the coupled equations. This kind of solution is not found in the usual nonlinear Schrödinger equation but is known to be a possibility in coupled systems of nonlinear equations such as those describing birefringent fibers. If multi-hump solutions exist for the laser-BEC system as well, it should be possible to use known methods of nonlinear optics and to study the interaction between the different soliton-like formations to infer whether there will be mutual repulsion or attraction or whether they can propagate together. This would justify the numerical results and provide an insight into the possibility of using these effects to manipulate localised atom-light structures. In what follows we will briefly review the basic coupled model and stationary coupled equations as well as the limitations imposed by a semi-classical description. We will then search for multi-hump solutions and study in particular the tail interaction of double-peaked solutions to show how, within this model, it is possible to expel solitary-like structures in a jet-like fashon, or even having them colliding and merging together into a single central structure.
Model equations. - The semi-classical coupled stationary equations as introduced in $[4,6]$ are given by

$$
\begin{array}{r}
\hbar \omega_{a} \Phi=\hat{H}_{0} \Phi+\left[U_{0} \Phi^{2}-\frac{\alpha}{4} \frac{\mathcal{E}^{2}}{\left(1-\frac{4 \pi}{3} \alpha \Phi^{2}\right)^{2}}\right] \Phi, \\
\nabla^{2} \mathcal{E}+k_{L}^{2}\left(1+\frac{4 \pi \alpha \Phi^{2}}{1-\frac{4 \pi}{3} \alpha \Phi^{2}}\right) \mathcal{E}=0 .
\end{array}
$$

where for the full atom wave function and the full laser field it has been assumed

$$
\begin{array}{r}
\Psi(\mathbf{r}, t)=\Phi(\mathbf{r}) \exp \left(-i \omega_{0} t\right), \\
\mathbf{E}(\mathbf{r}, t)=\operatorname{Re}\left[\mathcal{E}(\mathbf{r}) \exp \left(-i \omega_{L} t\right)\right] .
\end{array}
$$

Here, $\alpha(\omega)=-d^{2} / \hbar \Delta$ is the atomic polarizability at the laser frequency $\omega_{L}=k_{L} c$, with $\Delta=\omega_{L}-\omega_{a}$ being the detuning from the nearest atomic resonance frequency $\omega_{a}$, and $d$ is the dipole matrix element of the resonant transition. Besides, for the gas density we have $n=|\Psi|^{2}$, $U_{0}=4 \pi \hbar^{2} a_{s} / m, m$ is the atom mass and $a_{s}$ is the $s^{-}$ wave scattering length (which will be assumed positive as for repulsive atom-atom interactions) and $\hat{H}_{0}$ is the linear single-particle Schrödinger Hamiltonian. Furthermore, in order to reduce Maxwell's equations to three scalar equations, it has been assumed that $L_{n} \gg \lambda_{L}$ and $\nabla \epsilon \cdot \mathbf{E} \simeq 0$ ( $L_{n}$ is the characteristic length scale of transverse density modulations and $\lambda_{L}$ is the radiation wavelength). Notice that the atom equation is the approximate version of what was presented in [3], valid only under the assumptions of a semi-classical approach which limits the model to a well defined range of parameters: The concept of force being purely classical, quantum fluctuations, stochastic heating and any incoherent process are to be neglected which is a consistent assumption if large detunings are considered $|\Delta| \gg \omega_{a}, \Gamma$ ( $\Gamma$ is the natural line width of the atoms). Although these limitations are quite strict, the model should elucidate the basic physics of the interaction and hopefully the resulting structures will be resilient enough to be interesting even under non ideal conditions.

Finally, for a mean field model to be valid for the atom wave function, we must consider not only the zero temperature limit but also a low density limit with $n a_{s}^{3} \ll 1$, see [1]. Furthermore, a low density regime is required in order to avoid the singularity of the model and concomitant spurious collapse-like phenomena. In view of this limitation and in order to simplify the analysis, in what follows we will neglect the denominator in both equations. This is a delicate step and cannot be taken when analysing the coupled dynamics of the system, since it is not guaranteed that during the evolution the peak values of the atom density will satisfy the low density assumption (as seen in numerical simulations of the coupled evolution, [4], the nonlinear focusing action of the laser could be strong enough as to focus the atoms to very high peak densities in a single spot thus breaking the low density assumption). However, it is possible to accept this simplification 
when studying the stationary solutions with the proviso that only low density solutions will be accepted.

The physical setup of the problem. - In general, if laser and atoms are prepared in an initial state which is not a fully stationary state of the system, we expect the system to evolve showing changes in both the atom wave function and the laser amplitude profile with the propagation variable (hereafter chosen to be $z$ ). Thus it was observed in [6] and [7] via numerical simulations of the coupled equations that, depending on the parameters of the initial state, the propagation effects could have different outcomes. In particular, starting from a super-Gaussian laser amplitude much wider than the initial Gaussian atom density distribution, a regime of parameters (peak laser intensity and peak atom density) could be found for which the initial single-peak atom wave function and the flat laser amplitude profile slowly changed into two symmetrical peaks. Once these structures were formed, further propagation led to different possible scenarios: (1) outward motion of the two peaks moving farther apart from each other (this is what we call "jet emission"); (2) inward motion of the two peaks resulting in coalescence into a single central peak; or (3) inward motion of the two peaks resulting into a bound state with the two structures oscillating about the central position. Thus, from simulations of the coupled propagation equations it seems possible to see the creation of such symmetrical double-hump structures which however do not survive for long and undergo their own motion. In order to understand their nature and destiny (can they be thought of as solitary waves? Can we describe the outcomes of their coupled propagation in terms of soliton-soliton interactions?), we need to know what sort of stationary structures the model admits and work out the properties of their interactions.

Stationary solutions. - The existence of stationary solutions and their stability is fundamental from the point of view of realisable structures. We are interested in stationary solutions corresponding to mutual guiding in the form of long distance localised beam propagation. To analyse these solutions we focus on modes that are localised in the transverse direction, the analogue of Kerr spatial solitons, by assuming

$$
\begin{array}{r}
\mathcal{E}(\mathbf{r})=a\left(\mathbf{r}_{\perp}\right) \exp \left(i h_{a} z\right) \mathbf{e} \\
\Phi(\mathbf{r})=\phi\left(\mathbf{r}_{\perp}\right) \exp \left(i h_{\phi} z\right)
\end{array}
$$

where $\mathbf{r}_{\perp}$ denotes the dimension transverse to the propagation direction $z$, $\mathbf{e}$ is the polarization vector of the electric field, $a, \phi$ are real amplitudes and $h_{a}, h_{\phi}$ are the laser and atom propagation constants respectively. These solutions must satisfy the fully stationary equations derived from (1) and (2), which when written in normalised variables become:

$$
\begin{array}{r}
\tilde{\nabla}_{\perp}^{2} \tilde{\phi}-\beta \tilde{\phi}^{3}+s \tilde{a}^{2} \tilde{\phi}-2 \mu^{2} \kappa_{\phi} \tilde{\phi}=0 \\
\tilde{\nabla}_{\perp}^{2} \tilde{a}+3 s \tilde{\phi}^{2} \tilde{a}-2 \kappa_{a} \tilde{a}=0 .
\end{array}
$$

The normalisation used is: $\tilde{\mathbf{r}}=\mathbf{r} k_{L}$, for the atom wave function $\tilde{\phi}=\phi / \phi_{*}$ with $(4 \pi|\alpha| / 3) \phi_{*}^{2}=1$, for the laser $\tilde{a}=a / a_{*}$, with $m|\alpha| a_{*}^{2} /\left(2 \hbar^{2} k_{L}^{2}\right)=1, s=\operatorname{sign}(\alpha)$, and $\beta=6 a_{s} /\left(k_{L}^{2}|\alpha|\right)$ is directly proportional to the strength of the collisional nonlinearity. The tilde will be dropped hereafter unless otherwise stated. Furthermore, $\mu=k_{a} / k_{L}, k_{a}=\sqrt{2 m \omega_{a} / \hbar}, \kappa_{\phi}=\left(h_{\phi}^{2} / k_{a}^{2}-1\right) / 2$ and $\kappa_{a}=\left(h_{a}^{2} / k_{L}^{2}-1\right) / 2$. For simplicity, we will consider only one transverse dimension, $\mathbf{r}_{\perp}=x$ and assume $\mu=1$. To allow for mutual trapping, we will also assume $s=+1$.

A further change of variables allows to rewrite these equations in terms of the relative wave number only

$$
\begin{array}{r}
\bar{\phi}^{\prime \prime}+\left(\bar{a}^{2}-1\right) \bar{\phi}-\beta \bar{\phi}^{3} / 3=0, \\
\bar{a}^{\prime \prime}+\left(\bar{\phi}^{2}-\bar{\kappa}\right) \bar{a}=0 .
\end{array}
$$

where $\bar{\kappa}=\kappa_{a} / \kappa_{\phi}$ is the relative wave number, $\bar{x}=$ $\tilde{x} \sqrt{2 \mu^{2} \kappa_{\phi}}, \bar{a}^{2}=\tilde{a}^{2} /\left(2 \mu^{2} \kappa_{\phi}\right), \bar{\phi}^{2}=3 \tilde{\phi}^{2} /\left(2 \mu^{2} \kappa_{\phi}\right)$. Again the bar will be dropped hereafter.

To find localised solutions, we can solve this set of equations numerically as an eigenvalue problem. A first integral of motion of (10) and (11) can be obtained by multiplying (10) by $\phi^{\prime}$ and (11) by $a^{\prime}$ and integrating over $x$. Combining the two resulting integrals we find

$$
\phi^{2}+a^{\prime 2}-\phi^{2}-\kappa a^{2}+\phi^{2} a^{2}-\frac{1}{6} \beta \phi^{4}=\text { constant },
$$

The constant is zero for a localized solution vanishing at infinity. From this, we can deduce a relation between the values of $\phi$ and $a$ at the symmetry point $x=0$ where we require $\phi^{\prime}(0)=a^{\prime}(0)=0$ :

$$
\phi_{0}^{2}=\frac{3}{\beta}\left(a_{0}^{2}-1-\sqrt{\left(a_{0}^{2}-1\right)^{2}-2 \kappa a_{0}^{2} \beta / 3}\right),
$$

where $a_{0}=a(x=0), \phi_{0}=\phi(x=0)$. Then $a_{0}$ can be used as a shooting parameter to search for localised solutions with the shooting method [13]. For a fixed value of the eigenvalue $\kappa$, a value is assumed for $a_{0}$ with $\phi_{0}$ consequently calculated from (12) and a solution is calculated with an ordinary differential equation solver. The value of $a_{0}$ is than varied until a solution with the desired characteristics is found (single-hump, double-hump ...). As shown in our previous work [4], this equation admits single hump soliton-like solutions, in particular, it was shown that for one-scaled distributions $(\kappa=1)$ the set of eqs. (10), (11) reduces to one equation if $a(x)=\sqrt{\beta / 3+1} \phi(x)$, the soliton solution corresponding to $a_{0}=\sqrt{2}$ as is evident when solving the resulting nonlinear Schrödinger equation. We have expanded the family of solutions of (10), (11) and it is now clear that this set of equations admits multi-hump and periodic solutions as well for which the Hamiltonian (12) is exactly equal to zero. This can be easily seen in the simple case of $\beta=0$. At $\kappa \neq 1$ with $a_{0}=\sqrt{2}$, the coupled equations admit periodic solutions, see for instance fig.1 

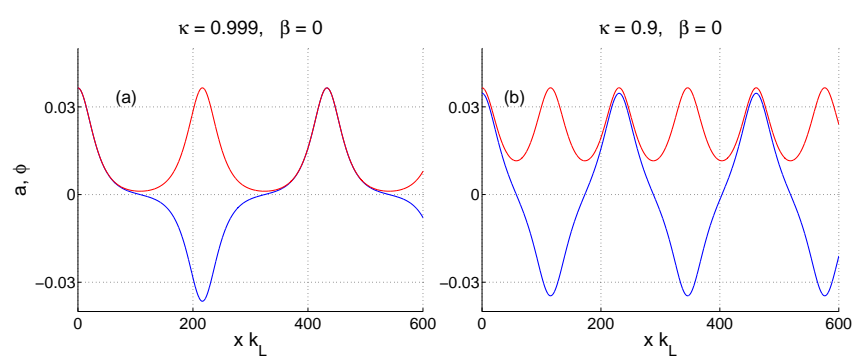

Fig. 1: Periodic solutions for $\beta=0$ at different values of $\kappa$. Blue line $\tilde{\phi}(x)$, red line (always positive) $\tilde{a}(x)$. All quantities normalized as in the text. For $\kappa>1$ the role of laser and atoms is simply inverted. Here $\kappa_{\phi}=10^{-3}$. Colour online.

Multi-hump solutions can be found as well, fixing $\kappa$ and varying $a_{0}$. Some examples for $\beta=0$ are shown in fig.2(a) and (b) while part (c) and (d) show examples of different multi-hump solutions for $\beta \simeq 38$ corresponding for instance to a detuning of 100 times the decay rate for ${ }^{87} \mathrm{Rb}$ atoms (the same value used in the previous numerical simulations). By varying the shooting parameter $a_{0}$ it is possible to find solutions with different number of peaks, thus it seems indeed possible to find multi-hump solutions for any value of $\beta$.

Jet emission. - We would like to underline the fact that these kind of multi-peak structure was never clearly seen in numerical simulations of the coupled propagation equation. As mentioned in the sectiom "The physical setup of the problem", we mainly observed the generation of two symmetric peaks (or four at higher initial atom densities) thus we cannot say anything about the generation of many-peak solutions within the range of parameters we have explored (the main limit being low atom densities). It is very likely that they can be generated at higher atom densities but appear only as a transient to transform quickly into more stable structures with two peaks only. This has obvious consequences for what could be oserved experimentally therefore it is important to unravel the dynamics of the structures. In particular we shall study the simplest case of two symmetric structures as the model case for this kind of dynamics. A knowledge of how they evolve gives the only acceptable indication of what could be observed. Therefore, once the existence of multihump solutions has been established, we can proceed to study the existence of stationary solutions that originate the ejected jets. The simplest case found in numerical simulations, [7], pictures an initially centrally localised bunch of atoms coupled to a localised profile of laser intensity (a Gaussian and a super-Gaussian respectively in the simulations), splitting into two "jets" which are then ejected and start to propagate in opposite directions together with two laser jets. The aim now is to find stationary solutions consisting of only two symmetric (or antisymmetric) localised humps. If they exist, we expect a tail-tail interaction between them because of the nature of the equations. In
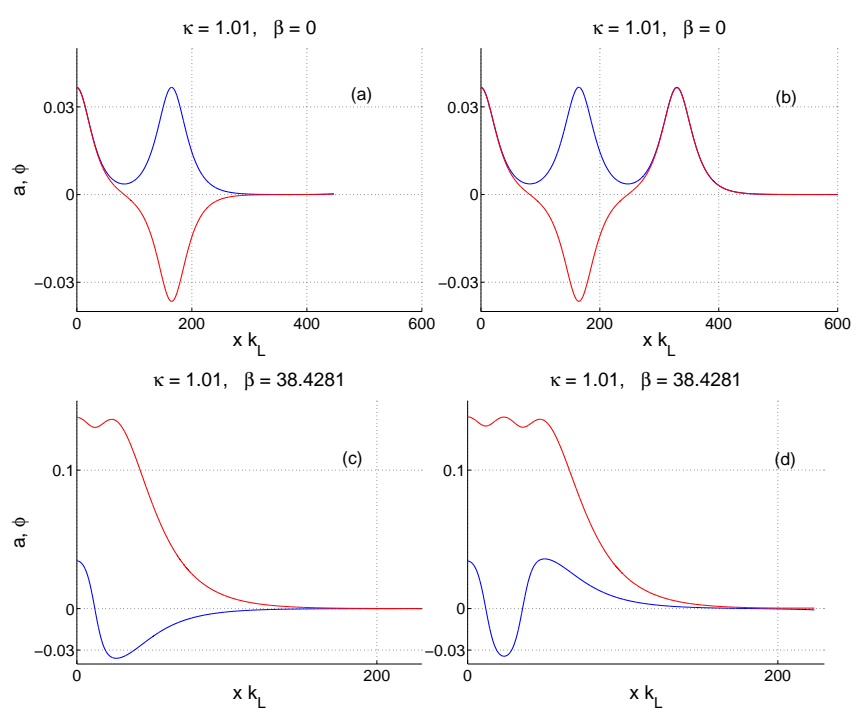

Fig. 2: Multi-hump solutions at $\kappa=1.01, \beta=0$ [(a) and (b)] and $38.4281[(\mathrm{c})$ and $(\mathrm{d})]$. Blue line (always positive in (a) and (b)) $\tilde{\phi}(x)$, red line (always positive in (c) and (d) $\tilde{a}(x)$. All quantities normalized as in the text. Shooting on the peak laser amplitude $a_{0}$ it is possible to identify different solutions with increasing number of peaks. Here $\kappa_{\phi}=10^{-3}$. Colour online.

the case of well separated humps, i.e. weak overlap and consequently weak tail interaction, a perturbative analysis completely akin to that introduced by Yang in [14] can be used to make predictions about the fate of the two humps. The question is whether they will propagate together or whether they will repel or attract each other because of their interaction.

An example of a symmetric two-peak solution is shown in fig. 3 for fixed $\beta$ and $\kappa$. These structures are found by imposing $\phi(0)=a^{\prime}(0)=0$, using the Hamiltonian (12) to obtain an expression for $a(0)^{2}=\phi^{\prime}(0)^{2} / \kappa$ and shooting on the value of the first derivative $\phi^{\prime}(0)$. Due to the symmetry of the equation, it is enough to calculate one half of the solution, say for $x>0$ and any combination (laser symmetric-atoms antisymmetric, laser antisymmetric-atoms symmetric and so on) is still a solution.

With a known double-peak solution showing two very well separated peaks, it is possible to study the nature of the interaction between the tails of the two peaks. We have followed the procedure by Yang in [14], who introduced a perturbative analysis of the interaction of vector solitons which we shall only briefly recall here. Modifying the perturbative approach of Karpman and Solov'ev, Yang found dynamical equations for the parameters of two-vector solitons weakly interacting with each other because widely separated. In particular, for cases in which the two components have phase difference 0 or $\pi$, Yang determined an equation for the dynamics of the vectorsoliton separation $\Delta x$ in the form of a Newtonian equation of motion $m d^{2} \Delta x / d z^{2}=-\nabla V$. The fixed points of 


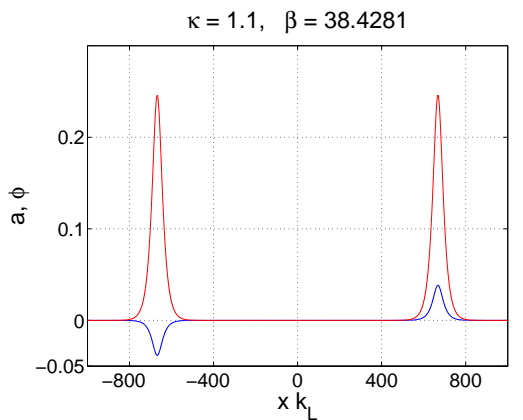

Fig. 3: An example of a double-hump solutions at $\kappa=1.1$ with $\beta=38.4281$. Blue line $\tilde{\phi}(x)$ (antisymmetric curve at lower amplitude), red line $\tilde{a}(x)$ (symmetric curve at higher amplitude). All quantities normalized as in the text. Here $\kappa_{\phi}=10^{-3}$. Colour online.

this equation give the separation distance of the two components of a stationary solution. We can therefore apply Yang's formulation to the main case under analysis, that of a two-peak stationary solution as the one numerically evaluated and shown in fig.3. This solution corresponds to what seen in numerical simulations of the atom-laser coupled propagation (see the section "The physical setup of the problem") before the two peaks start to move either inward or outward. With a detuning of about 100 times the decay rate for ${ }^{87} \mathrm{Rb}$ atoms (i.e., $\beta \simeq 38$ ), we find $\mathrm{a}$ peak atom density of $2.4910^{19} \mathrm{~m}^{-3}$ and a peak laser intensity of $0.051 \mathrm{~mW} / \mathrm{cm}^{2}$. Utilizing Yang's formula, we arrive for the case shown in fig. 3 at $\Delta \tilde{x}_{0}=1.334410^{3}$ which compares quite well with the separation found numerically $1.335910^{3}$. Therefore we can use the potential $V$ to infer the dynamics of the separation $\Delta x$. Again for the case shown in fig. 3 and using Yang's formula for $V$, the potential is shown in fig.4. It is clear that if the two components are generated at a distance $\Delta \tilde{x}<\Delta \tilde{x}_{0}$, the two solitons will repel each other and be ejected away whereas two components generated at $\Delta \tilde{x}>\Delta \tilde{x}_{0}$ will attract each other with the possible creation of a bound state where the two components oscillate about their equilibrium position. This model, based on the existence of multi-peaks solutions of our stationary equations, thus explains quite well the qualitative features of the numerical results of [7], both the jet emission and the formation of a bound state. It thus seems possible to say that the dipole-dipole interaction can lead to the generation of two-peak structures for both atoms and laser but the destiny of the two peaks is that of moving apart from each other in a solitary-like fashion or to move towards each other. This is indeed what seen via numerical simulations of the coupled propagation and we interpret the two-peak solutions as the unstable seeds of the evolution predicted by Yang's model and observed numerically.

Conclusions. - Numerical simulations of copropagating laser-BEC systems seem to indicate that not only

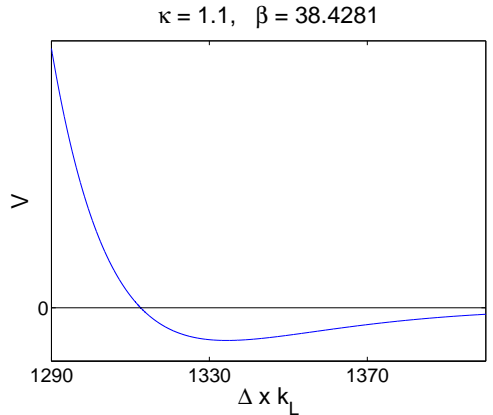

Fig. 4: Yang's potential $V$ versus the soliton separation $\Delta \tilde{x}$ for $\kappa=1.1$ and $\beta=38.4281$.

mutually localised structures can be formed, but also that solitary-like wave packets can be emitted out of the central interaction region. In order to understand the physics of the jet ejection, we have used a simplified system of coupled equations and studied the existence of possible multi-peaked stationary solutions, usually not possible for the nonlinear Schrödinger equation. Applying the results of a perturbative method elaborated in nonlinear optics to study their dynamics, we have found that jet emission and bound state formation can be explained by the tail interaction of the soliton components of a two-peak structure within the model we are using.

\section{REFERENCES}

[1] Leggett A.J., Rev. Mod. Phys., 73 (2001) 307.

[2] Saffman M., Phys. Rev. Lett., 81 (1998) 65.

[3] Krutitsky K.V., Burgbacher F. and Audretsch J., Phys. Rev. A, 59 (1999) 1517; Krutitsky K.V., Marzlin K.P. and Audretsch J., Phys.Rev.A, 65 (2002), 063609.

[4] Cattani F., Anderson D., Kim A. and Lisak M., JeTP Lett., 81 (2005) 561; Cattani F., Geyko V., Kim A., Anderson D. and Lisak M, Phys. Rev. A, 81 (2010) 043623.

[5] Cohen-Tannoudu C., Dupont-Roc J., Grynberg G., Atom-Photon Interactions (Wiley, Berlin) 1998.

[6] Cattani F., Kim A., Anderson D. and Lisak M., J. Phys. B: At. Mol. Opt. Phys., 43 (2010) 085301.

[7] Cattani F., Kim A., Anderson D. and Lisak M., [arXiv:1008.3302v1],Phys. Rev. A, 83 (2011) 013608.

[8] Wright E.M., Heatley D.R. and Stegeman G.I., Phys. Rep., 194 (1990) 309.

[9] Gubbels M. A., Wright E.M., Stegeman G.I., Seaton C.T. and Moloney J.V., J. Opt. Soc. Am. B, 4 (1987) 1837.

[10] Assanto G., Minzoni A.A., Peccianti M. and Smyth N.F., Phys. Rev. A, 79 (2009) 033837.

[11] Rodas-Verde M.I., Michinel H. and Perez-Garcia V.M., Phys. Rev. Lett., 95 (2005) 153903.

[12] Ostrovskaya E.A., Kivshar Y.S., Chen Z. and Segev M., Opt. Lett., 1999 (24) 327.

[13] Press W.H., Teukolsky S.A., Vetterling W.T. and FlanNery B.P., Numerical Recipes (Cambridge Univer- 
F. Cattani et al.

sity Press, New York) 2007, pp.956-961.

[14] YAng J., Phys. Rev. E, 64 (2001) 026607. 